\title{
La inteligencia militar como actor fundamental en el afianzamiento de los escenarios de paz*
}

| Fecha de recibido: 31 de enero del 2020 | Fecha de aprobación: 29 de abril del 2020 |

\author{
Abdón Estibenson Uribe Taborda \\ Magíster en Seguridad Operacional, \\ Magíster en Ciencias Militares Aeronáuticas \\ Comando Aéreo de Combate n. ${ }^{\circ} 5$ \\ Grupo de investigación: CETAD \\ Rol de investigador: intelectual \\ https://orcid.org/0000-0003-4058-4365 \\ $\triangle$ abdon.uribe@fac.mil.co
}

\section{Leonardo de Jesús Mesa Palacio}

Magíster en Sistemas Automáticos de Producción

Servicio Nacional de Aprendizaje (SENA) Grupo de investigación: CETAD

Rol de investigador: intelectual https://orcid.org/0000-0002-4903-723X $\triangle$ lomesap@sena.edu.co

\footnotetext{
* Artículo de reflexión.
}

Cómo citar este artículo: Uribe Taborda, A. E., \& Mesa Palacio, L. J. (2020). La inteligencia militar como actor fundamental en el afianzamiento de los escenarios de paz. Revista Ciencia y Poder Aéreo 15(1), 87-107. https://doi.org/10.18667/cienciaypoderaereo.656 


\section{La inteligencia militar como actor fundamental en el afianzamiento de los escenarios de paz}

Resumen: El presente documento de revisión resume el reto estratégico, operacional y táctico que tiene la inteligencia militar en Colombia, en el escenario del posconflicto. Por posconflicto se entiende el escenario posterior a la firma del acuerdo de paz entre el Gobierno colombiano y el grupo exguerrillero de las Fuerzas Armadas Revolucionarias de Colombia (FARC), convertido hoy en el partido político Fuerza Alternativa Revolucionaria del Común. El presente trabajo, basado en las experiencias vividas por países como Irlanda del Norte y El Salvador en tiempos de posconflicto, compara el proceso de dichos países con el escenario colombiano. En este escenario, se presenta la reactivación de actores generadores de violencia, un incremento de la delincuencia, la reactivación de grupos paramilitares y la formación de nuevos grupos al margen de la ley, también llamados Grupos Armados Organizados (GAO). Estos nuevos retos se deben afrontar usando la inteligencia militar, la cual se constituye en un actor fundamental en el afianzamiento de los escenarios de paz, en especial, en las zonas más apartadas y marcadas por la violencia de nuestro país. Este afianzamiento convertirá a esas zonas en parte del desarrollo competitivo de Colombia.

Palabras clave: Actividades delictivas; inteligencia aérea; posconflicto.

Abstract: This research summarizes the challenges for Colombian Air Intelligence and its application at the strategic, operational, and tactical levels during the post-conflict stage resulting from the peace process signed between the Colombian Government and the FARC guerrilla group. This study is based on the experiences of countries such as Northern Ireland and El Salvador, which went through various stages after signing a peace agreement for ending the conflict they sustained, which, like the Colombian case, left consequences of violence such as crime, the persistence of paramilitary groups, and the formation of new groups outside the law, among others. These challenges must be faced through military intelligence in order to combat any possible scourges and leverage the aim of the State of reaching the remote regions of the country stroke by violence and make them part of the competitive development model required in Colombia.

Keywords: Criminal Activities; Air Intelligence; Post-conflict.

Resumo: 0 presente documento de revisão resume o desafio estratégico, operacional e táctico que tem a inteligência militar na Colômbia, no cenário pós-conflito. Por pós-conflito entende-se o cenário após a assinatura do acordo de paz entre o governo colombiano e o grupo ex-guerrilheiro das Forças Armadas Revolucionárias da Colômbia (FARC), que hoje é o partido político Fuerza Alternativa Revolucionaria Común. Este trabalho, baseado nas experiências de países como a Irlanda do Norte e El Salvador em tempos de pós-conflito, compara o processo desses países como o cenário do pós-conflito colombiano. Neste cenário, são apresentadas a reativação dos atores geradores de violência, o aumento da delinquência, a reativação de grupos paramilitares e a formação de novos grupos fora da lei, também denominados Grupos Armados Organizados (GAO). Estes novos desafios devem ser enfrentados com a inteligência militar, que constitui um ator fundamental na consolidação de cenários de paz, especialmente nas áreas mais remotas e marcadas pela violência no nosso país. Essa consolidação fará dessas áreas parte do desenvolvimento competitivo da Colômbia.

Palavras-chave: Atividades criminosas; Inteligência aérea; Pós-conflito. 


\section{Introducción}

Cada conflicto, proceso y escenario de paz es único para cada nación. Sin embargo, en ellos se encuentran características generales que pueden suministrar información, así como experiencias y lecciones aprendidas que logran ser referentes para procesos similares en otros países.

El proceso actual de posconflicto en Colombia presenta variables y aspectos que difieren de los presentados por los procesos que otras naciones han experimentado en el establecimiento una paz permanente. De dichos aspectos, se destaca la existencia de otras organizaciones alzadas en armas. Entre ellas se encuentran las disidencias de las Fuerzas Armadas Revolucionarias de Colombia (FARC), las bandas criminales (Bacrim), la guerrilla del Ejército de Liberación Nacional (ELN) y grupos organizados ilegales que en la actualidad se dedican a la extorsión, el secuestro y la minería ilegal -también llamados Grupos Armados Organizados (GAO) - (Reyes, 2016).

Tomando como referente las vivencias de naciones como El Salvador e Irlanda del Norte, se establecen puntos de convergencia en la finalización de sus conflictos armados. Los grupos insurgentes dejaron a su paso secuelas de violencia, como víctimas inconformes, delincuencia, grupos paramilitares, nuevos grupos al margen de la ley, pobreza y desigualdad social. La forma en que las naciones afrontaron sus procesos de paz, se convierten en la base de análisis de las medidas y acciones necesarias para mejorar la respuesta de los entes de seguridad del Estado colombiano.

Los entes de seguridad del Estado son los garantes del bienestar y la soberanía, según el mandato constitucional. Ellos hacen un aporte activo en la toma de decisiones sobre las operaciones en contra de crímenes o el combate de fenómenos o amenazas. Estas decisiones, para el desarrollo de las operaciones militares, se basan en información esencial de las unidades de inteligencia que, "utilizando todos medios humanos o técnicos para la recolección, análisis y difusión de información" (Congreso de la República de Colombia, 2013), coadyuvan a proteger los derechos humanos, a prevenir y combatir amenazas.

\section{Problema}

Después del acuerdo de paz firmado en Colombia, se inició el establecimiento del escenario de paz. Algunos autores hablan de "un proceso largo, difícil y duro para el país" y lo consideran "el escenario adecuado para trabajar por una paz firme y duradera” (González, 2017 , p. 6). Sin embargo, para establecer un escenario de paz permanente, es necesario superar los nuevos retos que están surgiendo, como el de enfrentar a las disidencias y otros actores violentos al margen de la ley. Históricamente, la inteligencia militar ha desempeñado un importante papel en la identificación y combate de estas amenazas, lo que la convierte en un actor fundamental para la seguridad y defensa nacional. Según Barbosa, "la inteligencia militar es una importante herramienta para las fuerzas militares y el Estado en la toma de decisiones, garantizando la protección de los ciudadanos de diversas amenazas" (2016, p. 1).

En el presente documento se revisará y evaluará la participación histórica de la inteligencia militar de otras naciones en escenarios de posconflicto. Con esto se espera determinar cómo sus habilidades y acciones podrían ser un referente para el proceso de posconflicto que vive Colombia.

\section{Justificación}

Considerando las condiciones en las que se han desarrollado los conflictos armados y los escenarios de paz de otras naciones, es claro que los eventos de violencia posteriores a la firma de acuerdos de paz no son iguales a los fenómenos que vive actualmente Colombia. En el caso nacional, se cuenta con herramientas importantes para proponer mejoras en las capacidades de inteligencia militar y evitar los errores cometidos por otros países (Rojas, 2016, p. 62).

En países como Irlanda del Norte, gracias al aporte de la inteligencia británica, los grupos de inteligencia militar "lograron destacarse en el mundo gracias a la eficacia de sus operaciones militares, las cuales, 
condujeron al acuerdo de paz" (Bowlin, 1999, p. 8). Este caso, aunado al ejemplo de El Salvador, país que no se ha destacado en el mundo por sus capacidades de inteligencia militar, permite considerar diferentes escenarios que ofrecen información importante con respecto al uso de la información, el reconocimiento y las capacidades de vigilancia. Estas actividades, propias de la inteligencia militar, "se necesitan para sacar adelante las etapas criticas de un país" (Barbosa, 2016).

\section{Antecedentes}

La construcción de la paz se refiere al "conjunto de acciones que permite a una sociedad gestionar, prevenir y resolver el conflicto, a través de sus propias capacidades, sin recurrir al uso de la violencia" (IECAH, 2010). Colombia ha recurrido a procesos de paz realizados mediante negociaciones dialógicas, las cuales han resuelto algunos conflictos internos desde 1982 (Chernick, 1996). En el gobierno de Belisario Betancur (1982-1986) se buscó la apertura político-democrática con las guerrillas. Esta apertura condujo a un acercamiento con distintos grupos armados (como el Movimiento 19 de abril $-\mathrm{M}-19-\mathrm{y}$ las FARC). El acercamiento con el M-19 concluye con la toma del Palacio de Justicia, el 6 de noviembre de 1985. Después de la toma, se retoman los diálogos de paz y el 9 de marzo de 1990, en el gobierno de Virgilio Barco (1986-1990), se firma el acuerdo de paz entre el M-19 y el Gobierno colombiano. Esta firma puso fin a esta lucha armada y dio paso a la participación política del grupo político Alianza Democrática M-19, la cual se consolidó como fuerza política dentro del marco legal colombiano. Finalmente, se da una transformación de fondo en la política colombiana cuando dicho grupo político impulsa la Asamblea Nacional Constituyente, que tiene como resultado la Constitución Política de 1991 (Bernal, 2016). Más adelante, en 1991, el Gobierno Nacional también logra un pacto de paz, que contiene varias garantías políticas, con el Ejército Popular de Liberación (EPL), que era uno de los grupos guerrilleros más dogmáticos y beligerantes del país.
El Gobierno colombiano, actuando en concordancia con la Constitución Nacional que le exige la protección de los conciudadanos, ordenó realizar bombardeos contra la guerrilla de las FARC insistiendo en la necesidad de reducir los hombres en sus filas y de que el conflicto tuviera una terminación real. En la década de 1990 hubo un crecimiento militar y una expansión territorial con la que las FARC pasó de tener 48 frentes y 5800 combatientes en 1991, a tener 62 frentes y 28000 combatientes en el 2002. El grupo armado tenía una presencia en 622 municipios, es decir, en un $60 \%$ de los municipios del país (Centro Nacional de Memoria Histórica, 2013, p. 162).

Después de varios intentos fallidos de diálogos de paz con las FARC, la insurgencia de mayor capacidad delictiva firmó un acuerdo de paz con el Gobierno colombiano el 24 de noviembre del 2016 (Villarraga, 2017). Es importante mencionar que, aunque se logró firmar un acuerdo de paz con ellos, aún hace falta lograr un pacto de paz definitivo con el ELN y disolver de forma completa las estructuras paramilitares de las Autodefensas Unidas de Colombia (AUC), parcialmente desactivadas entre 2003 a 2006 con el proceso de paz firmado en Santafé de Ralito, Córdoba, el 15 de Julio del 2003 (Villarraga, 2017).

Después del compromiso de paz con las FARC, la tarea del Gobierno es construir una paz firme y duradera. En cumplimiento de esta labor, el Gobierno nacional debe hacer frente a los temas pendientes que han sido mencionados, sumando a estos los rezagos que deja el conflicto armado con las FARC, el incumplimiento de los acuerdos firmados y otras amenazas que pueden surgir en este escenario de paz. Durante este tiempo, el Gobierno Nacional tendrá que contar con el apoyo de sus instituciones militares, en especial la inteligencia aérea, la cual desempeña un papel importante en la toma de decisiones y el mantenimiento de una paz estable y duradera (Villarraga, 2017).

A lo largo de su historia, Colombia ha acumulado experiencias de diferentes acuerdos de paz entre los gobiernos y la mayoría de las guerrillas. Esto muestra los esfuerzos gubernamentales para construir la paz, pero también, que no se han podido controlar las tensiones y dinámicas de prolongación del conflicto (Barbosa, 2016). 


\section{Fundamento teórico}

Con el acuerdo de paz con las FARC se dan varios logros importantes que hoy permiten hablar de un escenario de paz. Uno de ellos, el cese del conflicto que, aunque permite reducir las afectaciones de la violencia en el país, no elimina por competo el accionar violento de ese grupo, el cual es uno de los rezagos del conflicto armado. Actualmente se combaten las disidencias de las FARC para garantizar la seguridad nacional. Este es uno de los principales desafíos para el Estado colombiano y las Fuerzas Militares (Villarraga, 2017).

En el nuevo escenario, como parte del afianzamiento de la paz en Colombia, se requiere realizar operaciones militares específicas. En estas operaciones, instituciones como la Fuerza Aérea Colombiana deben diseñar un conjunto de acciones que les permitan fortalecer sus capacidades de gestión de crisis con el fin de prevenir nuevos conflictos y crear condiciones para una paz duradera. Las habilidades complementarias que se deben desarrollar para afrontar los nuevos desafíos y retos de la seguridad nacional, deberán cumplir con el marco constitucional y legal que determina el accionar de las instituciones. Para conocer las cualidades que deben tener las operaciones de inteligencia aérea colombiana, es indispensable entender de dónde vienen las características y los retos que suponen las nuevas amenazas. Se requiere, igualmente, un entendimiento amplio de la importancia que tienen las operaciones militares en la toma de decisiones. Para esto se hace necesario conocer algunos conceptos relevantes, asociados al tema de la presente investigación (Barbosa, 2016).

\section{Inteligencia}

La inteligencia es definida como "la capacidad para pensar y para desarrollar el pensamiento abstracto, como capacidad de aprendizaje y de adaptarse a situaciones nuevas, o para solucionar problemas" (Mayer, 1983). En el contexto militar, es considerada como un elemento necesario en la proyección y la ejecución de las operaciones militares (Prieto del Val, 2014).
En las funciones de comando, el concepto se refiere a que cada área de comando debe disponer de la inteligencia requerida para la toma de decisiones (Llop et al., 2013).

En el marco legal colombiano, la Ley estatutaria 1621 del 17 de abril del 2013, capítulo 1, artículo 2, afirma que la función de inteligencia y contrainteligencia es "aquella que desarrollan los organismos especializados del Estado de orden nacional, utilizando medios humanos o técnicos para la recolección procesamiento, análisis y difusión de información”. La ley 162, por su parte, indica cómo proteger los derechos humanos "prevenir y combatir amenazas internas y externas contra el régimen democrático, constitucional y legal”, preservar la seguridad y la defensa nacional.

\section{Inteligencia militar}

La inteligencia militar se ha logrado posicionar como un instrumento requerido para planificar diferentes actividades tanto operacionales como administrativas. Su eficacia puede demostrar un amplio conocimiento sobre las actividades que el enemigo actual o potencial puede desarrollar. La inteligencia militar es necesaria para obtener la victoria que garantice la prevalencia de la democracia y la visión de una sociedad que está en constante cambio (Sainz, 2012).

La inteligencia militar es "el producto resultante de recolectar, evaluar e interpretar la información disponible que ofrece valor inmediato o potencial para el planeamiento y conducción de operaciones militares". La inteligencia militar abarca inteligencia de combate, inteligencia básica, inteligencia estratégica y la contrainteligencia.

En el sistema de inteligencia militar español, se habla de tres tipos de inteligencia:

- Estratégica: centrada en el plano político, sus receptores son el Gobierno y el Jefe del Estado Mayor de la Defensa (JEMAD), que sirve como enlace entre las decisiones políticas y las militares.

- Operativa: destinada al planeamiento, ejecución y apoyo de operaciones, sirve al mando de operaciones y los cuarteles generales. 
- Táctica: tiene en cuenta el terreno, meteorología, adversario, y responde a los fines inmediatos de las unidades sobre el terreno.

\section{Inteligencia militar en tiempos de paz}

Kent indica que la aplicación de la inteligencia "es tan real [...] en tiempos de paz como en tiempos de guerra" y, además, que la utilización de las capacidades de la inteligencia militar durante el desarrollo de una guerra y en época de paz reflejan consecuencias similares. "Los errores en la gran estrategia de la paz podrán no producir el espectáculo de un campo de batalla innecesariamente desastroso, pero a veces producen algo mucho peor" (Kent, 1950, p. 93).

En cuanto al caso colombiano, el señor mayor general Paredes indica que, en el periodo de "postconflicto", la estrategia militar debe "poner a disposición de sus comandantes los medios para prevalecer, diseñar los objetivos, evaluar las limitaciones del alcance del concepto de victoria y argumenta que no se debe perder el esfuerzo del poder militar" (Paredes, 2011, p. 7).

\section{Fuerza Aérea Colombiana en un escenario de paz}

El escenario de paz que vive actualmente Colombia obliga a las instituciones militares, como a la Fuerza Aérea Colombiana, a transformar su estructura organizacional para tener la capacidad de actuar bajo estándares internacionales y asumir los nuevos retos que trae consigo el posconflicto. Las instituciones deben ser especializadas y efectivas en su actuar, tener un mayor control de recursos de tipo técnico, económico y humano, aumentar la velocidad de respuesta y simplificar los procesos que ayuden a contrarrestar las amenazas (Fuerza Aérea Colombiana, 2016).

\section{Amenazas para el escenario de paz en Colombia luego de firmado el acuerdo con las FARC}

Antes de haberse dado la firma del acuerdo de paz, algunas fuentes afirmaban que Colombia no había logrado llegar a un acuerdo, porque su conflicto era muy diferente y las condiciones colombianas involucraban múltiples actores y fuentes de violencia (Chernick, 1996). Sin embargo, con los resultados de los últimos años, Colombia logró demostrar que, a pesar de sus particularidades, no era un caso excepcional, que podía aprender de otras experiencias y llegar a una solución negociada (Chernick, 1996). Ahora bien, como afirma Flavin (2003), "la terminación de conflictos es el final de la lucha pero no es el fin del conflicto" (p. 112). Este autor indica, además, que el objetivo de las operaciones militares es establecer las condiciones que obliguen a los beligerantes a poner fin a las hostilidades. Estas operaciones dependen del liderazgo militar y civil. Hay que reconocer, en todo caso, que el final del conflicto es tan crítico como la propia guerra. No obstante, este escenario también posibilita el reordenamiento territorial, al igual que el replanteamiento de nuevas formas de resolver las dificultades nacionales (Torres, 2016).

Los constantes ataques por parte de la guerrilla del ELN y las disidencias de las FARC ponen a prueba la estabilidad de la paz en Colombia. Aunque no se conoce una cifra exacta de las personas que hacen parte de estos grupos, las Fuerzas Militares calculan que el número supera las 1200. Estos grupos cuentan con un conjunto de mandos medios con capacidad bélica, presencia territorial, pretensiones políticas que siguen vigentes y una alianza con economías criminales (principalmente del narcotráfico). Estas condiciones ponen en riesgo la seguridad nacional (Álvarez et al., 2018).

Se puede observar que el Estado colombiano no tiene una completa presencia en lugares que se caracterizaron en el pasado por la presencia guerrillera. Esto favorece la aparición de grupos criminales emergentes que se componen de combatientes que no se desmovilizaron de la guerrilla, e incorporan adicionalmente a carteles tradicionales que aprovechan estas áreas huérfanas para establecerse y promocionar sus actividades delictivas migratorias (Estrada et al., 2016).

Los acontecimientos posteriores a la firma de la paz con las FARC indican que el Estado colombiano debe adaptarse a amenazas delincuenciales complejas. Estos hechos "obligarán a modificar sus políticas 
preventivas, los objetivos y funciones de sus sistemas de inteligencia para conseguir unas respuestas más flexibles y adaptables a las amenazas, obligando, en algunos casos, a modificar su percepción de seguridad" (Pulido, 2017).

Los temas de seguridad en Colombia, tras haberse realizado un acuerdo de paz, "han producido un debate en relación a la necesidad de la actualizar los procedimientos, medios y capacidades, tanto de las Fuerzas Armadas como las policiales, para adaptarse al nuevo escenario" (Pulido, 2017).

Pulido (2017) indica que las nuevas "actividades delictivas de las bandas criminales emergentes (Bacrim) plantean el pensamiento de nuevos retos a la Seguridad del Estado, en materia de los nuevos actores que intervendrán" (p. 1).

Por su parte, Molano, quien estudió los fenómenos más importantes en materia de seguridad, evolución y propuestas para la transformación de la Fuerza Pública colombiana en el posconflicto, indica que a corto plazo se debe dar un despliegue de batallones ligeros a los territorios colombianos con el fin de "dar respuesta inmediata a una posible mutación del crimen en los territorios de estabilización con presencia de las FARC" (Molano, 2016, p. 1). Un segundo componente a mediano plazo es el uso de la inteligencia militar y de policía para estabilizar las posibles amenazas a la seguridad de la nación (Molano, 2016).

\section{El papel de las fuerzas militares frente a las amenazas}

La reforma de la Fuerza Pública para afrontar el proceso de posconflicto, se enmarca en las reestructuraciones de seguridad como principal objetivo para proteger a la población. Se considera la posibilidad de establecer una Policía Civil Internacional (Civpol) bajo las órdenes de la onu en condiciones de no guerra, e igualmente se analiza el tamaño de las fuerzas militares en posconflicto, la capacitación y el reentrenamiento de estas.

Strong y Jaramillo (2014) ponen en evidencia el rol de la Fuerza Aérea en la doctrina de la Acción Integral como política de afianzamiento de la Seguridad
Democrática. Los autores analizan la doctrina, la labor del poder aéreo y la manera en que la Fuerza Aérea ha contribuido a la seguridad colombiana. Además, indican la importancia del poder aéreo, en el futuro, para el control territorial, puesto que se presentarán nuevas amenazas y el territorio nacional deberá estar preparado para la lucha contra el crimen, el tráfico ilegal, la piratería y la extracción ilegal de recursos naturales.

Por otra parte, Esquivel (2016) expone, en cinco apartados, el análisis histórico de la Fuerza Aérea Colombiana entre 1998 y el 2015. El artículo da a conocer las estrategias que ha utilizado la Fuerza Aérea en sus operaciones para contribuir al cese del conflicto interno colombiano. Este trabajo es pertinente para la investigación aquí planteada, ya que aborda todas las operaciones realizadas por la Fuerza Aérea durante el conflicto interno colombiano, desde 1998 hasta el 2015, cuando el Gobierno ordenó cancelar los bombardeos contra las FARC (este fue el punto de partida para los acuerdos con el grupo revolucionario).

\section{La inteligencia militar como herramienta fudamental para la toma de decisiones}

Siguiendo con el tema de la inteligencia, y para tener una visión más clara de su importancia en periodos de pos-guerra y paz, Campbell (2013) explora las causas y los cambios que ha sufrido la inteligencia desde el final de la guerra fría. Indica que la inteligencia en la actualidad es un asunto de todos. Esto se ve reflejado en las zonas de conflicto en donde se recoge información de inteligencia, la cual es utilizada y analizada por mandos superiores e inferiores. Otro ejemplo es el de los ciudadanos comunes y corrientes, quienes se han convertido en productores y consumidores de inteligencia. Este público espera que las áreas de inteligencia y seguridad de los países sean proactivas y colaboradoras para evitar las amenazas y esperan que los gobiernos mantengan sus libertades civiles.

Para conocer un poco sobre el origen y desarrollo de la inteligencia en Gran Bretaña, Charters (2009) nos muestra cómo la inteligencia surgió como una parte central de la teoría y la práctica de la contrainsurgencia británica en la época de posguerra. Hoy en día, 
la inteligencia es ampliamente reconocida en las operaciones y campañas contra la insurgencia. Pero no siempre fue así. El artículo de Charters es importante para la presente investigación porque establece el desarrollo de la inteligencia británica y analiza cómo la experiencia operativa de la posguerra cambió la doctrina antes de la guerra, a través del ensayo y el error en las condiciones únicas de Irlanda del Norte. Este artículo sostiene además que la inteligencia permite que las fuerzas de seguridad se anticipen a los problemas para prevenirlos. La prevención depende de una recolección y análisis eficaz de la información.

Monroy habla de la importancia de los diálogos de paz en Irlanda del Norte a partir de la firma del acuerdo del Viernes Santo de 1998. Este acuerdo ha servido como guía para solucionar los conflictos armados en otras latitudes (Monroy, 2017). El documento analiza la historia del conflicto armado del Irlanda del Norte, el acuerdo de Viernes Santo y sus principales consecuencias para el posconflicto. Analiza además las consecuencias del conflicto que deben solucionarse en el periodo siguiente a la firma del acuerdo de paz. El conflicto irlandés se ha convertido en un referente de análisis obligatorio para la solución de conflictos como el colombiano. Según Monroy, la contribución de los servicios de inteligencia es importante para negociar con los grupos insurgentes.

Colombia ha usado operaciones de inteligencia militar para combatir las fuerzas insurgentes a lo largo del conflicto armado. Entre el 2007 y el 2015 se realizaron bombardeos de precisión, ayudados por la inteligencia previa, que debilitaron a los grupos ilegales. De esta manera se "crearon las condiciones para atacar a los líderes medianos y altos” (Spencer \& Gómez, 2011, p. 107). Igualmente, Esquivel (2016) se refiere a estas como "operaciones decisivas que garantizaron el debilitamiento de sus oponentes".

Pulido (2017) nos muestra el concepto de "inteligencia híbrida como nueva forma de adaptación de las estrategias tradicionales contrainsurgentes" (p. 56). El objetivo de la inteligencia híbrida es realizar un análisis de las nuevas políticas de seguridad e inteligencia que Colombia aplicará en la etapa posterior a la firma del acuerdo de paz para contrarrestar la contrainsurgencia y los nuevos fenómenos criminales que buscan el control territorial (Pulido, 2017). El artículo menciona que, después de la firma del acuerdo de paz, se observa la necesidad de transformación de la Fuerza Pública para que sea competente en el escenario de posconflicto. Este artículo es pertinente para la presente investigación puesto que destaca la importancia de la inteligencia colombiana en la etapa posterior al acuerdo de paz, la necesidad de prevenir la criminalidad organizada y la insurgencia criminal.

Barbosa (2016) explica "la importancia de la inteligencia militar a lo largo de la historia del conflicto colombiano" (p. 3). En su texto expone algunas labores que la especialidad de inteligencia puede cumplir en el contexto del posconflicto para que el Estado colombiano pueda garantizar la seguridad y defensa nacional.

A continuación, se presentan dos experiencias de inteligencia militar desarrolladas en el conflicto y en su etapa posterior con los grupos guerrilleros. Estas contienen elementos importantes que ayudarán a identificar cuáles son las capacidades que debe tener la inteligencia aérea colombiana en la toma de decisiones para el escenario de posconflicto.

\section{Irlanda del Norte}

Luego del acuerdo de paz, Irlanda del Norte inició una nueva etapa en la que persistieron varias problemáticas. Entre ellas, se destaca la legitimidad estatal, que, como afirman Lavaux \& Lair (2009), "ha permitido la presencia de grupos paramilitares" (p. 11). La falta de control estatal, después de firmado el acuerdo de paz, e incluso antes de su firma, condujo a la conformación de grupos disidentes que rechazaron los acuerdos. Igualmente, los líderes paramilitares que cambiaron sus actividades delictivas, lo cual generó un mayor índice de inseguridad y criminalidad. Las actividades criminales, económicamente lucrativas, compensaron las pérdidas que se dieron con el fin de la guerra. En este caso, varios grupos disidentes evolucionaron hasta conformar verdaderas mafias involucradas en 
cualquier tipo de tráfico criminal que les permitiera mantener su estilo de vida (poder, estatus, seguridad, ventajas económicas). Todos estos factores dificultaron los procesos de desmovilización y aumentaron la resistencia de los miembros de esas organizaciones, lo que hizo difícil la implementación de cualquier alternativa para neutralizar su reincidencia (Lavaux \& Lair, 2009).

Para contrarrestar la insurgencia y el terrorismo en la transición hacia un estado de paz en Irlanda del Norte, la comunidad de inteligencia británica se vio obligada a enfrentar sus mayores desafíos operacionales después de la segunda guerra mundial. Aunque muchas de las operaciones que llevaron a cabo fueron valientes e inventivas con éxito, al inicio sus operaciones fueron desorganizadas, más allá de la ley británica y adoptaron métodos que, en última instancia se hicieron, contraproducentes. La inteligencia británica jugó un papel crítico en la guerra en Irlanda del Norte y sus experiencias tienen hoy aplicaciones universales para cualquier servicio de inteligencia (Bowlin, 1999). Sin duda, en la guerra y en el posconflicto de Irlanda del Norte, la comunidad de inteligencia británica evolucionó en materia de tácticas y técnicas.

\section{Experiencia de la inteligencia británica en Irlanda del Norte}

En 1970, la comunidad de inteligencia británica se dividió en dos, unos apoyaron a la Unión Soviética y otros a Irlanda del Norte. Para los oficiales de inteligencia que actuaron en Irlanda del Norte, esta fue la oportunidad de probarse a sí mismos personal y profesionalmente. En este escenario hubo recompensas presupuestarias, las cuales se traducían en prestigio institucional. Según Mark Bowlin, a pesar de que la mayoría de las actividades de las organizaciones de inteligencia británica eran clasificadas (y por eso no han sido confirmadas al público) según la Ley de Secretos Oficiales, eso no es un obstáculo para bosquejar una imagen razonable de la comunidad de inteligencia británica y su historia reciente en Irlanda del Norte (Bowlin, 1999).

La participación de la inteligencia británica en la guerra de Irlanda del Norte tiene, como es lógico, una historia mixta de éxitos y fracasos. Sin embargo, estas organizaciones de inteligencia rara vez se dan el lujo de elegir o aceptar la aprobación pública. En consecuencia, se sabe poco sobre sus éxitos. Las fallas se hacen públicas con mayor frecuencia que el éxito y tienden a dar forma a la percepción de la eficacia que el público tiene de las operaciones de inteligencia (Bowlin, 1999).

Para comprender la coordinación y el desarrollo de las operaciones de inteligencia en Irlanda del Norte, es importante conocer cómo se encontraban integradas las diferentes agencias que intervinieron durante el conflicto armado y el escenario de paz.

\section{Agencias de inteligencia nacional}

Para esa época, el Reino Unido tenía tres agencias de inteligencia nacional: la Seguridad Servicio (MI5), el Servicio Secreto de Inteligencia (MI6) y el Gobierno Sede de Comunicaciones (GCHQ). Hubo dos eventos simultáneos, ocurridos en agosto de 1979, que sacudieron a la comunidad de inteligencia británica nacional. El primero de ellos fue el asesinato de Lord Mountbatten y el segundo fue la emboscada del Irish Republican Army (IRA) a una patrulla del ejército británico en Warrenpoint. En esta emboscada murieron dieciocho soldados. Estos eventos llevaron a los programas de inteligencia humana (Humint) a actualizarse y coordinarse de manera más eficiente. El exdirector del MI6, Maurice Oldfield, fue nombrado por el primer ministro británico y la ministra Margaret Thatcher para enderezar la situación de inteligencia en Irlanda del Norte. Oldfield recomendó al Gobierno que la inteligencia británica coordinara sus actividades a través de un conjunto regional de Grupos de Tareas y Coordinación (TCG). Se estableció un TCG en Castlereagh para coordinar las operaciones de Belfast; un segundo grupo en Armagh, que coordinó las actividades de inteligencia en el sur de Ulster, y un último grupo en Londonderry, para coordinar el norte de la provincia. Con esto, las unidades encubiertas ya no podían andar por ahí actuando de forma independiente, en su propio nombre y siguiendo sus propias agendas. Estas unidades eran responsables ante una unidad centralmente basada en TCG, la cual también coordinaba a otras unidades para 
trabajar juntas cuando se requirieran diferentes habilidades en un área de operación (Bowlin, 1999).

Cada TCG era dirigido por un oficial de la Rama Especial y tenía representantes de la inteligencia del Ejército asignados a él (oficiales de la MI5; Urban, 1992, p. 95). Además de la MI5 y el Mı6, la última agencia de inteligencia británica, era la responsable de la inteligencia de señales (SIGLNT). Esta es la contraparte de la Agencia de Seguridad Nacional (NSA). La idea de los TCG era mejorar dramáticamente la coordinación de inteligencia (Bowlin, 1999).

El GCHQ era la entidad encargada de la recopilación de información de otras agencias. Además, prestaba asesoría sobre procesos de investigación y recopilación de información en telecomunicaciones. La MI5 era la organización responsable de la recopilación de inteligencia doméstica y su responsabilidad principal era la contrainteligencia británica. Esta realizaba una función aproximadamente análoga a la función de contrainteligencia del FBI, aunque la MI5 carecía de la autoridad de aplicación de la ley. El MI6 era responsable de las operaciones de inteligencia extranjera (así pues, era análoga a la Agencia Central de Inteligencia, CIA). Tanto la MI5 como el MI6 se involucraron ampliamente en Irlanda del Norte y desde 1969 dedicaron un esfuerzo considerable para expulsar al otro de Irlanda. La opinión generalizada entre los integrantes de la M15 y el MI6 era que el IRA podría haber sido el adversario, pero el otro servicio era el enemigo (Bowlin, 1999).

\section{Inteligencia militar en Irlanda del Norte}

Según Urban, el gobierno del primer ministro, Edward Heath, sintió que el MI6 no solo tenía una historia más extensa en Irlanda, sino que también se adaptaba mejor al trabajo en campo, es decir, el establecimiento de redes de informantes (Urban, 1992). Sin embargo, en 1972 la MI5 logró convencer a Londres de que sus esfuerzos para proteger al Reino de los actos violentos requerían de su presencia en el otro lado del mar de Irlanda. Todo esto provocó una rivalidad entre los dos servicios, históricamente conocida como una desagradable guerra burocrática de territorio.

\section{Agencia de inteligencia de ejecución de la ley}

Otro evento importante para las fuerzas de seguridad de Irlanda del Norte y las operaciones de inteligencia que combatieron al IRA fue la implementación de una política conocida como Ulsterization, en 1977. La política reconocía que el conflicto debía ser civilizado, así que la primacía de la aplicación de la ley fue restablecida (Kennedy-Pipe, 1997). En consecuencia, se construyó el servicio de inteligencia de la Real Policía de Ulster (RUC), que estableció una red de informantes de inteligencia. Dicha red estaba conformada por jóvenes locales que conocían el terreno y crecieron en el mismo entorno que el IRA; comprendían la cultura única de Irlanda del Norte, y además tenían una estrecha relación con la policía nacional de la República de Irlanda (Bowlin, 1999). Sin embargo, debido a que el RUC era visto como simpatizante de los paramilitares leales, el ejército restringió frecuentemente los intercambios de inteligencia con el Ruc para evitar que la información llegara a los "hombres duros" protestantes (Gill, 1994, p. 157).

Establecer lazos de confianza entre las organizaciones especiales fue difícil y las acusaciones de abuso de prisioneros durante el interrogatorio ayudaron al fracaso de la inteligencia en ese momento. Algunas de las operaciones llevadas a cabo por la inteligencia británica fueron las operaciones secretas de cobro, operaciones de propaganda, penetración clandestina del IRA y participación de inteligencia en las llamadas operaciones "disparar a matar", entre otras (Bowlin, 1999).

En materia de inteligencia militar, se considera que, gracias a la efectividad de la inteligencia militar, hoy en día existen lecciones muy valiosas sobre cómo contrarrestar el terrorismo. Una de esas lecciones viene del "grado de organización y el enfoque que utilizaron a la hora de planificar la ofensiva" (Miranda, 2016). Como ejemplo, se destaca la resistencia del IRA y su adaptabilidad, pues "consiguió poner en jaque al Estado británico haciendo uso de su sofisticación y su capacidad de operar con precisión" (Miranda, 2016).

De igual manera, Gran Bretaña es considerada como un líder mundial en contrainsurgencia. Allí, la 
inteligencia surgió como una pieza central de la teoría y la práctica en la era de posguerra. Los británicos experimentaron una empinada curva de aprendizaje en inteligencia a nivel operacional y táctico que les permite tener la flexibilidad para adaptarse a condiciones locales específicas (Charters, 2009). Luego de mucho tiempo, las experiencias y cambios dentro de las instituciones de las cuales la inteligencia hace parte, lograron consolidar a una institución importante para quienes viven un conflicto o un periodo de posguerra (Charters, 2009).

A finales del siglo xIx, la inteligencia militar británica todavía estaba en su infancia. Existía un fuerte prejuicio contra la inteligencia, que prevaleció como un remanso profesional, solo adecuado para aquellos oficiales considerados no aptos para el comando. En consecuencia, el personal y las unidades de inteligencia se incubaron al final de un conflicto y luego se disolvieron o se vinieron abajo con su finalización. Por lo tanto, el apoyo de inteligencia a menudo era insuficiente (Charters, 2009).

Los servicios secretos tuvieron un comienzo aún menos auspicioso y una existencia igualmente tenue antes de la Segunda Guerra Mundial. El Servicio de Seguridad y Secreto de Inteligencia (MI5 y MI6) estableció dos organizaciones de inteligencia simultáneamente, en 1909, como respuesta a la amenaza de espionaje alemán (Charters, 2009).

El ejército británico entró intelectualmente desprevenido contra la insurgencia en la posguerra. En consecuencia, a menudo fue superado por sus enemigos en esta época crucial de la guerra. La combinación de terrorismo y propaganda emuló conscientemente el modelo del IRA y su política confundió a los responsables de la formulación de políticas, a los comandantes militares y a las propias fuerzas de seguridad británica (Charters, 2009).

Hay quienes sugieren que la inteligencia británica fue en última instancia muy efectiva, pero a costa de emplear algunos métodos muy dudosos. "Hoy tuvimos mala suerte, pero recuerda que solo tenemos que tener suerte una vez" (Bamford, 2005, p. 3). Se emplearon controvertidas técnicas de interrogatorio británicas, conocidas como las "cinco técnicas", como ayuda para el interrogatorio en Irlanda del Norte en el otoño de 1971 (Newbery, 2009). Se empleaban técnicas como la privación del sueño, limitación de la dieta a pan y agua, la aplicación de ruidos para causar desorientación, cubrimiento de la cabeza con una capucha, mantener al interrogado de pie con las manos levantadas y contra la pared durante prolongados periodos de tiempo. Según un comité del ministerio del interior, se consideró que estas técnicas estaban justificadas en excepcionales circunstancias, como las que atravesaba Irlanda del Norte, en las que "la obtención de información resultaba vital” (Alonso, 2001, p. 154).

Bennett (2010) argumenta que la estrategia militar británica fue adaptable, alternó entre medios defensivos y ofensivos dependiendo de los cambios del contexto político. La política de bajo perfil permitió al ejército consolidar una base firme para operaciones ofensivas contra el Ejército Republicano Irlandés Provisional (PIRA). Dicha política se consideró exitosa, porque contrasta con la violencia republicana, para negociar y comprometerse cuando sea necesario. La campaña británica en Irlanda del Norte, a principios de la década de 1970, a menudo se presenta como consistente en su carácter represivo. Su fracaso o éxito militar se relacionaron con fines políticos.

La experiencia británica en Irlanda del Norte ha servido como modelo para el ejército de los Estados Unidos en la preparación y realización de "operaciones de establecimiento de la paz", aunque sus operaciones de pacificación han requerido de diferentes entrenamientos, diferentes equipos y enfrentan hechos diferentes. Sin una extensa previsión y preparación, el empleo de fuerzas entrenadas convencionalmente para ejecutar el establecimiento de la paz podría ser inconveniente (Miller, 1993, p. 41).

Henriksen (2008) proporciona una excelente reconstrucción histórica para enmarcar su análisis del conflicto y las formas de operar del IRA y el ejército Británico. Henriksen se ha orientado por la búsqueda de ideas sobre la destreza de la contrainsurgencia en Irlanda del Norte para aplicarlas y mejorar las fuerzas combatientes actuales de los Estado Unidos.

Irlanda del Norte es un modelo viable o válido para el mundo de hoy. Pero el lector debe tener 
cuidado al sacar conclusiones rápidas. Es necesario abordar algunos problemas subyacentes. El más importante es el papel del gobierno del Reino Unido. El Reino Unido es una democracia rica, capaz, moderna y liberal, con un sistema estatal y una sociedad civil bien desarrollados.

\section{Experiencia del conflicto en El Salvador}

La historia de El Salvador inicia con la crisis general de 1930, producida por la gran depresión de la economía internacional. El régimen político de El Salvador fue incapaz de responder a esta crisis, que va desde 1931 hasta 1980 y se caracterizó por algunos factores socio-económicos como la pobreza en que estaba viviendo la población, la creciente desigualdad social y la concentración de riqueza (Ramos \& Cordoba, 2007).

Desde 1929, El Salvador ha tenido varios golpes militares así como gobiernos de extrema derecha. Todo esto dio origen a la conformación de grupos guerrilleros, apoyados por movimientos de izquierda, que impulsaron el nacimiento del Frente Farabundo Martí para la Liberación Nacional, el 10 de octubre de 1980 (Global, 2013).

\section{Las fuerzas militares durante el conflicto armado en el El Salvador}

El Salvador inició su conflicto armado en 1980, año en que estalla la guerra civil que enfrenta a las Fuerzas Armadas de El Salvador (FAES) y las fuerzas insurgentes del frente Farabundo Martí para la Liberación Nacional (FMLN). Este conflicto es considerado como uno de los más sangrientos que ha visto el hemisferio occidental. Se estima que durante esta guerra, que duró doce años, murieron más de cien mil personas.

El doctor Corum indica que las Fuerzas Armadas en su conjunto tenían graves dificultades de liderazgo. Además, la selección para el mando se basaba más en las conexiones políticas y los patrocinadores que en los méritos. La mayoría de los enlistados eran simples hombres reclutados (o presionados), el entrenamiento era mínimo y mediocre. En síntesis, "era un ejército que no estaba preparado para una guerra seria" (Corum, 1998, p. 28).

En comparación con otras áreas de sus fuerzas militares, la Fuerza Aérea Salvadoreña (FAS) fue el brazo de servicio más profesional. Era una pequeña fuerza formada por un pequeño "batallón de paracaidistas, una unidad antiaérea y cuatro pequeños escuadrones con un total de 67 aviones; [fue] considerada como la principal fuerza de combate del fAS" (Corum, 1998, p. 32).

La FAS tenía dos bases aéreas principales. La primaria estaba en llopango, en la parte sur del país; la otra, un poco más pequeña, se ubicaba en San Miguel (Corum, 1998).

\section{El Salvador después de la guerra}

El fin de la guerra, en 1992, no significó la disminución del crimen. En el periodo de guerra, murieron anualmente alrededor de 6250 personas. En el periodo de la posguerra, los homicidios pasaron de 8019 en 1996 a 8281 en 1998. "El Salvador, con una proliferación de bandas delictivas tras la guerra civil (1980-1989), se ubicó en el primer lugar de la lista, seguido de Irak, donde la invasión de Estados Unidos iniciada en marzo del 2003 se convirtió en un conflicto caracterizado por ataques entre musulmanes chiítas y sunitas, sin ser considerado una guerra civil" (Periódico Desde Abajo, 2011).

Los causantes de estos hechos violentos, fueron aquellos que intervinieron en el conflicto armado, pues una vez terminado se relacionaron con las redes de delincuencia común. De este modo, se presentaron altas tasas de criminalidad y de delincuencia debido a dificultades en la reinserción económica de los excombatientes, el vacío institucional en materia de seguridad pública, justicia y la existencia de armamento circulante que quedó en manos de civiles después de finalizado el conflicto.

Después de doce años de guerra, se firmó la paz y se aplicó una reducción de las Fuerzas Armadas, que pasaron de tener 56000 miembros a 20000 (Pérez, 
2009). También, con los acuerdos de paz, se incluyeron reformas del sector militar y la seguridad pública (Montes, 2005). Las fuerzas militares se limitaron únicamente a "la defensa de la soberanía y la integridad del territorio nacional" (Montes, 2009), lo que implicó medidas para la "reducción, reestructuración y depuración militar” (García, 2014). Los acuerdos relacionados con las Fuerzas Armadas implicaron la reducción de tropas y cambios en la doctrina en materia de inteligencia (García, 2014).

Cale afirma que el acuerdo de paz firmado entre El Gobierno de El Salvador y el FMLN, intermediado por las Naciones Unidas, no pudo haber sido firmado sin la ayuda militar de los Estados Unidos, específicamente, del Grupo Auxiliar de Asesoramiento Militar (Cale, 1996). Este grupo se desplegó en El Salvador inmediatamente después de que la administración del presidente Carter tomó la decisión de apoyar al gobierno salvadoreño con asistencia económica y militar.

Un primer contingente de cincuenta y seis efectivos militares de Estados Unidos, desplegados en El Salvador para finales de marzo de 1981, se desglosó en las siguientes áreas funcionales:

a. Aumento del personal de militar (MilGroup) en la embajada de Estados Unidos.

b. Equipo de entrenamiento móvil (MTT) que trabajaba al interior del MilGroup para fines administrativos, logísticos y de comando.

c. Equipo Naval de Entrenamiento (NTT), que buscaba ayudar a la Armada salvadoreña a mejorar su capacidad para interceptar la infiltración de armas destinadas a las guerrillas de izquierda.

d. Personal de entrenamiento y mantenimiento de helicópteros.

e. Pequeños equipos de entrenamiento de unidades, de cinco hombres cada uno. Estos equipos proporcionaban guarnición y entrenamiento a la nueva fuerza de reacción rápida de los salvadoreños.

f. Equipos especializados (OPAT), de cinco hombres cada uno, para ayudar a los cinco comandos regionales de El Salvador en la planificación de operaciones específicas.
En el grupo inicial se incluyeron soldados de las Fuerzas Especiales del Ejército que habían sido entrenados específicamente para ese tipo de operaciones. Algunos soldados de las Fuerzas Especiales (boinas verdes) también se encontraban en El Salvador para ayudar a capacitar al personal militar salvadoreño en comunicaciones, inteligencia, logística y otras habilidades. Estas capacitaciones estaban diseñadas para mejorar sus capacidades de interceptación de la infiltración y de responder rápidamente a los ataques terroristas (Montgomery, 1982).

\section{Bases legales}

La Constitución política de Colombia (1991), artículo 217 , es el punto de partida dentro del marco legal colombiano que rige las actividades de inteligencia y contrainteligencia para el proceso de posconflicto y construcción de la paz. En dicho artículo se indica que

la nación tendrá para su defensa unas fuerzas militares permanentes constituidas por el Ejército, la Armada y la Fuerza Aérea. Las fuerzas militares tendrán como finalidad primordial la defensa de la soberanía, independencia, la integridad del territorio nacional y el orden constitucional.

Es necesario considerar este apartado en la presente investigación, puesto que la Fuerza Aérea ejerce soberanía en el espacio aéreo de Colombia. Para ello, la institución debe hacerse responsable de la preparación de la fuerza para los operativos y ejecución de la guerra, así como de las operaciones militares en épocas de posconflicto y en tiempos de paz.

En este marco regulatorio, es importante también la Ley 684 del 2001 (del 18 de agosto del 2001), por la cual se expiden "normas sobre la organización y funcionamiento de la seguridad y defensa nacional y se dictan otras disposiciones" (Congreso de la República de Colombia, 2001). La inteligencia aérea militar debe contribuir, según esto, con la edificación de la paz en un periodo de posconflicto. 
Al hablar de posconflicto y de construcción de la paz, es necesario hacer referencia también a la Ley 1794 del 11 de julio del 2016, por medio de la cual se aprueba "el acuerdo marco entre las Naciones Unidas y el Gobierno de la República de Colombia relativo a las contribuciones al sistema de acuerdos de fuerzas de reserva de las Naciones Unidas para las operaciones de mantenimiento de la paz". El objetivo de esta ley es "establecer el marco de contribución del Gobierno colombiano a las operaciones de mantenimiento de la paz". Es importante aclarar que para las operaciones de mantenimiento de la paz de las Naciones Unidas el Gobierno colombiano proporcionará el equipo y el personal necesario. Dentro de este personal, se encuentran las unidades de la Fuerza Aérea que se requieran para mantener la paz y seguridad de la nación en busca de la paz. El personal debe ser capacitado con un alto nivel crítico y de inteligencia.

También se encuentra el Decreto 2176 del 2015, mediante el cual se reglamenta "el funcionamiento del consejo interinstitucional del Posconflicto creado por el artículo 127 de la Ley 1753 del 2015 por la cual se expide el Plan Nacional de Desarrollo 2014-2018, Todos por un nuevo país. El consejo interinstitucional articula los programas que contribuyen a la construcción de paz en el posconflicto.

Por último, se encuentra la Ley estatutaria 1621 del 17 de marzo del 2013, por medio del cual el Congreso de la República decreta la "normas para fortalecer el marco jurídico que permite a los organismos que llevan a cabo actividades de inteligencia y contrainteligencia cumplir con su misión constitucional y legal, y se dictan otras disposiciones". En el capítulo 1 de esta ley, el Congreso de la República de Colombia establece los límites, función, mecanismos de control, supervisión, protección de agentes y la finalidad de las actividades de inteligencia y contrainteligencia. La Fuerza Pública, y los organismos que indica la ley, son los encargados de realizar la función de inteligencia y contrainteligencia, por lo que deben cumplir adecuadamente con la Ley estatutaria 1621. El artículo 3 ilustra la pertinencia de la presente investigación, al referirse a "los organismos que llevan a cabo la función de inteligencia y contrainteligencia", dentro de los cuales se encuentra la Fuerza Aérea.

\section{Marco metodológico}

A continuación, se presentan los temas que se van abordar en el desarrollo metodológico. Para el cumplimiento de los objetivos propuestos, se realizó una investigación de tipo descriptivo con enfoque cualitativo.

\section{Alcance de la investigación}

La investigación fue de tipo descriptivo y utilizó herramientas cualitativas para la recolección de datos. Esta recolección se hizo por medio de una revisión de la literatura sobre las problemáticas que se pueden presentar en un país en la etapa de posconflicto y sobre cómo la inteligencia de la Fuerza Aérea de Colombia puede afrontar las diferentes etapas en los procesos de consolidación de la paz. Con esta información se buscó especificar las propiedades y características de los fenómenos, para someterlos al análisis y "medir o recoger información de manera independiente o conjunta sobre las variables a las que se refieren" (Hernández et al., 2004, p. 95).

\section{Diseño de investigación}

Para este trabajo de investigación se empleó el estudio de casos, pues este permite comprender los diferentes contextos investigados para la construcción de conocimiento sobre la base de conceptos con el fin de describir, verificar o generar contenidos. En este caso, se tomaron como referentes las experiencias vividas por otros países. Así, este estudio se basó en "una discusión en la literatura que aborda la investigación cualitativa, con respecto a la forma de considerar los estudios de caso" (Hernández et al., 2010).

La investigación cualitativa establece una postura con respecto al conocimiento existente del caso de estudio. La estrategia permite que el investigador 
cualitativo desarrolle un paradigma y un enfoque de recolección de información acerca de los eventos. Se abordan de este modo varios temas teóricos generales asociados con los casos estudiados (Hernández et al., 2010). La estrategia de investigación se orientó por hechos con antecedentes históricos relacionados, y resaltó la importancia de estudios previos sobre el tema, lo que permitió obtener las conclusiones pertinentes (Hernández et al., 2010).

\section{Presentación y análisis de resultados}

En este apartado se muestran los resultados y su respectivo análisis. Nos referiremos a las capacidades que debe tener la inteligencia aérea para afrontar los efectos venideros en la etapa del posconflicto.

\section{Resultados}

De acuerdo con el análisis cualitativo realizado, se encontró que existen vivencias en la etapa de posconflicto de otros países que pueden ser referente para la toma de decisiones en el área de inteligencia aérea en Colombia.

Entre las amenazas existentes de cara al posconflicto se encuentra la minería ilegal, que es uno de los problemas que afectan la seguridad y el medio ambiente. Otras amenazas frecuentes son la extorsión, el narcotráfico, el tráfico de armas, el crimen transnacional organizado, el terrorismo y el fenómeno de la convergencia. Esta última se refiere a la asociación de distintas amenazas según sus objetivos o sus medios. Todas estas actividades delictivas lucrativas deben tener un mayor control por el Estado colombiano (Ardila \& Torrijos, 2017).

De igual manera, en el posconflicto se presentan situaciones particulares como la de excombatientes que amenazan la paz o guerrilleros que no encuentran un papel en la vida política (Garzón et al., 2003, p. 19). Sumando a esto los fenómenos de globalización que determinan la futura economía mundial y afectan a los países en desarrollo de una forma significativa, surgen las denominadas nuevas amenazas. La actividad delictiva, criminal o terrorista no puede atacarse individualmente puesto que tiene un carácter global, de interconexión y sumatoria transnacional. Esto hace que los enfoques en materia de seguridad tradicionales queden desactualizados (Ardila \& Torrijos, 2017).

Dentro de estas nuevas amenazas se encuentran el terrorismo, el tráfico mundial de drogas, la delincuencia transnacional organizada, el tráfico ilegal de armas, la "trata de personas, los ataques a la seguridad cibernética, la posesión y uso de armamento de destrucción masiva y sus medios vectores por terroristas" (Ardila \& Torrijos, 2017).

En el territorio colombiano se manifiestan estas problemáticas después de la finalización de conflictos. Además, en nuestro país hay factores complejos como la inconformidad social; la presencia de actores ilegales que explotan los recursos naturales, lo que les da gran rentabilidad; las fronteras porosas, que permiten los fenómenos de convergencia y nuevas amenazas; la debilidad estatal; la corrupción, y el subdesarrollo (Ardila \& Torrijos, 2017).

Sumado a esto, algunos autores manifiestan que la inteligencia militar necesita cada vez más análisis de datos para pronosticar las demandas sociales, siempre crecientes, de seguridad (Velasco et al., 2012). Estas demandas provienen de las opiniones de los individuos y estas, a su vez, surgen de la base de información a la que el individuo tenga acceso. "Nadie puede tener una opinión sobre un libro cuya existencia desconoce" (Velasco et al., 2012). Las comunidades actuales tienen un amplio acceso a la información y comunicación, y basadas en ellas se hacen una idea de las situaciones y sucesos y se manifiestan con respecto a ellos. En definitiva, una desinformación puede causar diferencias de opinión que, a su vez, se convierten en un reto para la fuerza de cara a al posconflicto. En el área social, esto constituye un reto de trasformación de conductas y de opinión (Velasco et al., 2012).

La inteligencia aérea puede proporcionar datos para análisis con énfasis en al área social. Estos datos brindan conocimiento sobre las actividades que se están desarrollando y sobre la posible manipulación de la información para transformar la opinión cultural a 
favor de los grupos ilegales y continuar con sus actividades delictivas. Esta problemática se ha visto en otros campos con la utilización de abundantes tecnologías y medios para trasmitir información de forma masiva (Velasco et al., 2012).

En su cuaderno de trabajo, la doctora Carolina Sancho manifiesta lo importante que es pensar en la "ciberinteligencia" como un área en la que interactúan las "personas, organizaciones e instituciones". La ciberinteligencia es una realidad teórica que emerge para impulsar a las diferentes organizaciones de un Estado a prepararse para afrontar las variantes de trasformación culturales que ocultan el accionar de nuevas estructuras delictivas. "Un creciente uso del ciberespacio y constante aumento en su cobertura, puede verse limitado por amenazas y vulnerabilidades" (Sancho, 2018).

El ciberespacio es actualmente la última dimensión o dominio reconocido en la cual las personas, organizaciones e instituciones a nivel nacional, internacional y transnacional interactúan con la finalidad de comunicarse, intercambiar bienes o servicios, generar valor agregado a productos, entre otros (Sancho, 2018).

\section{Análisis de los resultados}

Al revisar los resultados obtenidos del análisis de la literatura consultada y de las vivencias de Irlanda del Norte y El Salvador, se pudo determinar que los escenarios posteriores al cese de la confrontación armada implican retos e interrogantes para los actores sociales. Igualmente, los alcances de los procesos hacen que estos acuerdos de paz puedan tomar un rumbo incierto (Herbolzheimer, 2012).

La disolución de un grupo guerrillero no implica necesariamente el fin de la violencia ni la terminación de los problemas relacionados con el enfrentamiento armado. Esto significa que la mayor parte de los retos del posconflicto estarán relacionados con dos tipos de amenazas: las amenazas existentes y las nuevas amenazas. Obligatoriamente, esto generará cambios en la seguridad que deberán ser la prioridad para el Estado colombiano y sus instituciones (Prieto del Val, 2014).

\section{Discusión}

El mayor general Paredes (2011) considera que un "país pacifista por naturaleza como Colombia, debe construir su pensamiento estratégico sobre Hipótesis de Guerra". Por esta razón, los grandes desafíos derivados de un entorno estratégico como el colombiano consisten en pensar en la reconfiguración de las amenazas a la seguridad y la defensa nacional. Esto implica la proyección del fortalecimiento y modernización de las capacidades existentes de inteligencia de la Fuerza Aérea en el corto, mediano y largo plazo (Paredes, 2011).

El fortalecimiento de la inteligencia aérea cumplirá "un papel decisivo en los escenarios donde se desarrolla el día a día de la etapa de posconflicto" (MINTA). La inteligencia aérea hará "presencia efectiva donde la requieran e indistintamente, en la aplicación de la fuerza o en apoyo de labores humanitarias, al contar con una capacidad de privilegio que la hace de por sí superior" (MINTA).

En ese sentido, se debe realizar una reingeniería a la inteligencia aérea "a fin de [que se especialice] en su rol operativo, liderando las operaciones aéreas, basada en los insumos obtenidos y procesados a través de un recurso humano competente y comprometido con el país y con la fuerza" (MINTA).

La inteligencia aérea

tiene su razón de ser en el conocimiento preciso de la amenaza real y potencial, para evaluarla, prever sus movimientos y anticiparse a su accionar; lo que le permitirá a la Fuerza Pública o a las agencias de seguridad del Estado, hacer más eficiente la aplicación de la fuerza cuando se considere necesario (MINTA).

En Suramérica, la investigación académica en inteligencia estratégica ha sido poco estudiada. Debido a los constantes cambios, que implican la aparición de fenómenos de mayor complejidad como los delitos trasnacionales, se requiere de una inteligencia dinámica y oportuna que logre poner en marcha planes directos para responder a estos nuevos retos (Bartolomé et al., 2016). La inteligencia estratégica militar es la encargada de prever y enfrentar los problemas de 
la sociedad ligados la seguridad y la defensa. En Suramérica, considerada como una región de paz pero con una historia de "altos niveles de violencia y fenómenos que por su complejidad requieren de lecturas cada vez más especializadas" (Bartolomé et al., 2016), la inteligencia estratégica militar debe ser capaz de "desentrañar las causas y ofrecer respuestas adecuadas para la toma de decisiones" (Bartolomé et al., 2016), en especial en las etapas posteriores al conflicto.

Latinoamérica es una de las áreas con menor índice de conflictividad entre los países. No existen armas nucleares en la región y hay un bajo índice de inversión en gasto militar. La óptica de la estrategia militar permite deducir que ningún país latinoamericano representa una amenaza para el orden mundial. Cobo (2014) afirma que Latinoamérica "es una región que tiene problemas de violencia de tipo regional, que no repercute con gran importancia políticamente hablando ante las grandes potencias mundiales". Latinoamérica es "una zona de gran potencial en la producción de materia prima para las industrias de países desarrollados" (Cobo, 2014).

\section{Conclusiones}

Como resultado de la investigación teórica presentada, se concluye que los procesos de conflicto y posconflicto no son nuevos para Colombia y muchos otros países. Han existido diálogos, acuerdos y firmas de procesos de paz con los grupos al margen de la ley gracias al apoyo y mediación de organismos internacionales; pero también por situaciones que los han obligado a determinar un cese al fuego, desarme, desmovilización y a hacer que los excombatientes se reintegren a la vida civil.

En el periodo posterior al conflicto, se deben instaurar nuevas estrategias que permitan combatir las nuevas actividades delictivas mediante la aplicación de las capacidades existentes de la inteligencia aérea colombiana. Estas capacidades se fortalecieron durante el conflicto vivido, y se requiere que se sigan fortaleciendo y se modernicen para contrarrestar las actividades delictivas que prevalecen. Aunque los procesos de paz y posconflicto son únicos y diferentes para cada nación, es importante que, en el momento de iniciar las actividades de inteligencia, se cuente con las herramientas necesarias y el personal capacitado que se enfoque en combatir la criminalidad de una manera acertada.

El fin de la guerra no se traduce en el fin del conflicto en la región. Esta es solo la oportunidad para que la Fuerza Pública pueda encauzar sus capacidades en el combate de las problemáticas que se derivan de la continuidad de la criminalidad. Los acuerdos de paz se convierten en oportunidades para que las naciones fortalezcan sus instituciones para que estas, a su vez, puedan responder a los nuevos retos. Estos retos tienen que ver con los grupos que siguen utilizando medios delictivos para mantener el control en las áreas donde anteriormente otros grupos delinquían. Enfrentar a estos grupos se convierte en un desafío esencial para la inteligencia militar en el posconflicto.

La firma de la paz, "no siempre produce los resultados esperados ni cumple las expectativas generadas por la negociación, a pesar de que la terminación de la guerra brinda un entorno positivo para la reducción de los niveles de violencia política" (Prieto del Val, 2014, p. 4). Los brotes de violencia e insurgencia "dependen de una serie de factores internos y externos no necesariamente vinculados a las negociaciones de paz" (Prieto del Val, 2014, p. 7), sino a la conformación de nuevas estructuras que persisten. Hay una mutación en la manera en que opera la criminalidad y el terrorismo transnacional. Estos exigen que la inteligencia militar tome un rol determinante y definitivo, siguiendo la premisa de que "la clave del poder aéreo está en la acertada selección de los objetivos y la clave de la selección de esos objetivos es la Inteligencia Aérea" (Prieto del Val, 2014, p. 11).

\section{Recomendaciones}

El presente trabajo de investigación sugiere adoptar las siguientes recomendaciones, emitidas con el fin de mitigar los brotes de violencia producto de actores siguen generando violencia en la clandestinidad. El fortalecimiento de la inteligencia aérea permitirá 
suministrar información detallada y en tiempo real para cumplir con los objetivos de interés nacional. Es necesario que la inteligencia y contrainteligencia aérea aporten información oportuna, profesional y consecuente con los deberes constitucionales y morales. La inteligencia aérea debe servir como garante de la seguridad e integridad de todos los colombianos, mediante desarrollo de las siguientes actividades:

- Tomar las medidas requeridas para "prevenir la ruptura de la cohesión interna de la guerrilla y asegurar que cualquier fragmentación o criminalización, no arruine las posibilidades de continuar con el proceso de reinserción a la vida civil" (Herbolzheimer, 2012).

- Erradicar la confrontación armada y construir un escenario que cuente con el compromiso necesario para las partes. Garantizar la oportunidad, el cumplimiento de los tiempos, el respeto de los actores y los alcances del proceso, para que el acuerdo de paz no tenga un rumbo incierto (Herbolzheimer, 2012).

- El posconflicto es el escenario adecuado para que el estado colombiano, en colaboración de las Fuerzas Armadas, utilice la inteligencia militar como garante para llegar sin obstáculos a las zonas más apartadas y marcadas por la violencia.

- Abordar los retos en la etapa de posconflicto por medio del conocimiento que se ha adquirido con las vivencias de otros países, las cuales brindan herramientas para la toma de decisiones.

- Aplicar los conceptos, las capacidades existentes y mejoras tecnológicas para vigilancia, control e inteligencia aérea, con el único fin de proteger al estado colombiano y cuidar su infraestructura energética y vial.

- Que la inteligencia aérea colombiana sea usada para consolidar los fines del Estado. Con la finalización de la guerra por la desmovilización de las FARC surgen nuevas necesidades de transformación por parte de la Fuerza Pública, de acuerdo con los cambios en el panorama nacional, que obligan a redefinir sus funciones y dispositivos.
- La aplicación de la inteligencia tendrá que adaptase a las nuevas etapas de la seguridad, que presentarán una serie de desafíos que deben ser advertidos desde la actualidad. No se debe esperar a que surjan nuevas amenazas sin que el estado colombiano esté preparado para ellas. Es esencial adoptar un enfoque diferenciado porque el postconflicto será muy diferente de una región a otra (Strong \& Jaramillo, 2014).

- Reducir el riesgo de reproducción de la violencia y de recaída en el conflicto. Para ello, será crucial contener el mercado de la violencia, bloquear las posibilidades y ocasiones de reincidencia de los excombatientes, y prevenir el resurgimiento de una facción insurgente o la persistencia de un remanente de las guerrillas que se margine del proceso y que insista en reivindicar la lucha armada, en conexión, probablemente, con actividades criminales (Molano, 2015).

- Se debe desarrollar una propuesta para las operaciones militares y de paz que sean necesarias, con el propósito de anticiparse a las amenazas que puedan venir en esta etapa de posconflicto. En este punto es importante el papel de la inteligencia, ya que no existe otra organización que conozca en mayor detalle a los grupos guerrilleros que han participado en el conflicto colombiano.

- Brindar capacitación y entrenamiento a las Fuerzas Militares, con énfasis en la mutación de la criminalidad. Adquirir tecnología de punta y otros elementos fundamentales para realizar efectivamente la labor constitucional de proteger la soberanía nacional (MINTA).

- La inteligencia aérea debe contar con "tecnología de punta, que detecte y neutralice el accionar delictivo de la amenaza según sea su lineamiento o conformación armada sea posible su neutralización" (MINTA).

- Se deben adquirir nuevos equipos técnicos de inteligencia y contrainteligencia con el fin de modernizar los equipos existentes. Se debe garantizar su reposición, actualización y el fortalecimiento de la inteligencia humana (MINTA). 
- Combatir el accionar de las amenazas existentes con tecnología de punta. En especial, debe enfrentarse la minería ilegal como uno de los mayores problemas de seguridad y medio ambiente. A este problema se suman amenazas frecuentes como el narcotráfico, la extorsión, el terrorismo y el fenómeno de la convergencia (Ardila \& Torrijos, 2017).

- Combatir las nuevas amenazas, que incluyen el narcotráfico internacional, la delincuencia transnacional organizada, el tráfico ilegal de armas y su conectividad, la trata de personas, los ataques a la seguridad cibernética, la posesión y uso de armamento de destrucción masiva, entre otras (Ardila \& Torrijos, 2017).

\section{Referencias bibliográficas}

Alonso, R. P. (2001). Irlanda del Norte: una historia de guerra y la búsqueda de la paz. Editorial Complutense S.A.

Álvarez, E., Pardo, D., \& Cajiao, A. (2018). Trayectorias y dinámicas territoriales de las disidencias de las FARC. Fundación Ideas para la Paz (FIP).

Ardila C. C., \& Torrijos R. V. (2017). Políticas públicas de seguridad y defensa: herramientas en el marco del postconflicto en Colombia. Escuela Superior de Guerra. https://doi. org/10.25062/9789585605497

Bamford, B. (2005). The role and effectiveness of intelligence in Northern Ireland. Intelligence \& National Security, 20(4), 581-607. https://doi.org/10.1080/02684520500425273

Barbosa, M. (2016). Desafios de la Inteligencia Militar del Ejército Nacional en un escenario de posconflicto. Universidad Militar Nueva Granada.

Bartolomé, M., Sancho, C., Maldonado, C., Pérez, J., Cruz, G., Pérez, C., Balbi, E., Velastegui, M., Rivera, F., Cabrera, A., \& Ordóñez, M. (2016). Inteligencia estratégica: perspectivas desde la región suramericana. Universidad de las Fuerzas Armadas ESPE. https://www.academia. edu/30787200/Inteligencia_Estrat\%C3\%A9gica_Contempor\%C3\%A1nea_ESPE-Ecuador_2016_

Bello-Montes, C. (2005). Posconflicto y desmovilización: comparativo de las experiencias en Colombia, Camboya y El Salvador. Revista Criminalidad. La nación y el crimen trasnacional, 232-241.
Bello-Montes, C. (2009). Un análisis del homicidio después del proceso de desmovilización de los grupos de autodefensa. Revista Criminalidad, 51(1), 163-177.

Bennett, H. (2010). From Direct Rule to Motorman: Adjusting British Military Strategy for Northern Ireland in 1972. Studies in Conflict \& Terrorism, 33(6), 511-532. https:// doi.org/10.1080/10576101003752648

Bernal, L. (2016). Proceso de paz del movimiento 19 de abril (M19) con el gobierno de Virgilio Barco. "Subvertir la paz, negociar la democracia” [Tesis de pregrado no publicada]. Universidad Distrital Francisco José de Caldas. http://repository.udistrital.edu.co/bitstream/11349/5859/1/Lizarazo\%20Bernal\%20Liseth\%20Andrea\%202017.pdf

Bowlin, M. (1999). British Intelligence and the IRA: The Secret War in Northern Ireland, 1969-1988. Naval Postgraduate School.

Cale, M. (1996). The United States Military Advisory Group in El Salvador, 1979-1992.

Campbell, S. H. (2013). Intelligence in the Post-Cold War Period. Intelligence; Journal of U.S. intelligence studies, 19(3), 45-52. https://www.afio.com/publications/CAMP BELL\%20Stephen\%20Part\%201\%20WinterSpring2013\% 20AFIO\%20INTELLIGENCER.pdf

Centro Nacional de Memoria Histórica. (2013). Los orígenes, las dinámicas y el crecimiento del conflicto armado. Centro Nacional de Memoria Histórica.

Charters, D. A. (2009). Counter-Insurgency Intelligence: The Evolution of British Theory and Practice. Journal of conflicts studies, 9, 55-74.

Chernick, M. (1996). Aprender del pasado: Breve historia de los procesos de paz en Colombia (1982-1996). Colombia Internacional, 36, 4-8. https://doi.org/10.7440/colombiaint 36.1996 .02

Cobo, I. F. (2014). Análisis crítico de la seguridad en Iberoamérica. http://www.ieee.es/Galerias/fichero/docs_analisis/2014/ DIEEEA29-2014_Seguridadlberoamerica_IFC.pdf

Colombia, Cp. (1991). Constitución política de Colombia. Legis. Congreso de la República de Colombia. (2001, 13 de agosto). Ley 684 del 2001. Diario Oficial 44.522. http://www. suin-juriscol.gov.co/viewDocument.asp?ruta=Leyes /1695676l

Congreso de la República. (2013, 17 de abril). Ley estatuaria 1621 del 2013. Diario Oficial 48.764. https://www.uiaf.gov. co/sistema_nacional_ala_cft/normatividad_sistema/ leyes/ley_estatutaria_1621_2013 
Congreso de la República. (2016, 11 de junio). Ley 1794 del 2016. Diario Oficial 49.931. https://diario-oficial.vlex. com.co/vid/ley-1794-2016-medio-645146137

Corum, J. (1998). The Air War in El Savador. Airpower Journal, 12(2) 27-44.

Esquivel, R. (2016). La Fuerza Aérea colombiana y el cese del conflicto armado (1998-2015). Revista Científica General José María Córdova, 14(17), 377-401.

Estrada, F., Moscoso, F. F., \& Andrade, N. (2016). Políticas de Seguridad contra el Narcotráfico. Análisis político, 86, 29(86), 3-34. http://dx.doi.org/10.15446/anpol.v29n86.58039

Flavin, W. (2003). Planning for Conflict Termination and Pos-conflict Success. Parameters, us Army War College Quarterly, 33(3), 95-112.

García, V. (2014). Reformas al sector seguridad en contextos de post-conflicto armado. Conferencia FLACSO-ISA. http:// web.isanet.org/Web/Conferences/FLACSO-ISA\%20Bue nosAires\%202014/Archive/6ce04630-f865-4f27-97462e6bbc953154.pdf

Garzón, J. D., Parra, A. D., \& Pineda, A. S. (2003.). El Posconflicto en Colombia:cordenadas para la paz [Tesis de pregado no publicada]. Universidad Jeveriana.

Gill, P. (1994.). Policing Politics: Security Intelligence and the Liberal Democratic State. Frank Cass.

Global, C. (2013, 8 de junio). El post conflicto de El Salvador: ¿una lección para Colombia? Blog de FM El Ciudadano Global. http://ciudadanoglobalfm.blogspot.com/2013/ 06/el-post-conflicto-de-el-salvador-una.html

González, A. (2017). Relación entre conflicto y posconflicto: Colombia y los acuerdos de paz. IEEE. http://www.ieee.es/ Galerias/fichero/docs_analisis/2017/DIEEEA25-2017_ Paz_Colombia_Postconflicto_AGM.pdf

Hernández, R., Fernández, C., \& Baptista, P. (2004). Metodología de la Investigación. McGraw-Hill Interamericana.

Henriksen, T. (2008). What Really Happened in Northern Ireland's Counterinsurgency: Revision and Revelation. JSOU Report 08-5. The Jsou Press.

Herbolzheimer, K. (2012). La paz: objetivo claro con rumbo incierto. Conciliation Resources. https://www.c-r.org/ es/news-and-views/comment/la-paz-objetivo-clarocon-rumbo-incierto

IECAH. (2010, 23 de noviembre). Construcción de la paz. IECAH. https://www.iecah.org/index.php/investigacioncp

Kennedy-Pipe, C. (1997). The Origins of the Present Troubles in Northern. Harry Hearder.

Kent, S. (1950). Inteligencia estratégica para la política mundial norteamericana. Pleamar.
Lavaux, S., \& Lair, E. (2009). Estado del arte, El conflicto en Irlanda del Norte. Facultad de Ciencia Política y Gobierno y de Relaciones Internacionales, Universidad del Rosario.

Llop M. S., Martínez, E. L., \& Valeriano E. F. (2013). Apuntes de inteligencia básica. División de publicaciones de la Escuela Superior de Guerra Naval.

Mayer, R. (1983). Pensamiento, resolución de problemas y cognición. Paidós Ibérica.

Miller, W. (1993, 19 de mayo). The British Experience in Northern Ireland: A Model for Modern Peacemaking Operations? [Manuscrito]. School of Advanced Military Studies.

Miranda, E. (2016, 18 de octubre). La estrategia contra el terror en Irlanda del Norte. elordenmundial.com.

Molano, J. L. (2016). Pautas de corto y mediano plazo para analizar la recomposición de la fuerza pública de cara a un eventual escenario de estabilización y posconflicto. http://www.pensamientopenal.com.ar/system/files/ 2016/05/doctrina43474.pdf

Molano R. A. (2015). El posconflicto en Colombia: Reflecciones y propuestas para recorrer la transición. Instituto de Ciencia Política Hernán Echavarría Olózaga.

Monroy, M. (2017). La transición de Irlanda del Norte del conflicto al posconflicto: guía para otras latitudes. InterNaciones, 10, 31-52. https://doi.org/10.32870/in.v4i10.6889

Montgomery, T. (1982). Revolution in El Salvador: Origins and Evolution. Colorado.

Newbery, S. (2009). Intelligence and controversial British interrogation techniques: the Northern Ireland case, 1971-2. Department of History, Trinity College Dublin.

Paredes, L. F. (2011). Seguridad y Estratégia: los desafíos del futuro. Revista Fuerzas Armadas, 217, 4-5.

Periódico Desde Abajo. (2011). El Salvador, Honduras y Colombia, entre los países más violentos de Latinoamérica. https://www.desdeabajo.info/mundo/item/1842 4-el-salvador-honduras-y-colombia-entre-los-pa\%C3 \%ADses-m\%C3\%A1s-violentos-de-latinoam\%C3\%A9ri ca.html.

Pérez, T. H. (2009). El papel de la democracia en sociedades posconflicto y en situación de conflicto armado interno. El caso de El Salvador y Colombia. Universidad de La Salle de Bogotá.

Presidencia de la República. (2015, 9 de noviembre). Decreto 2176 del 2015. https://bit.ly/2XqQg5w

Prieto del Val, T. (2014). La inteligencia militar, una constante histórica. Instituto Español de Estudios Estrategicos.

Pulido, J. (2017). La amenaza de la insurgencia criminal en Colombia: El concepto de inteligencia híbrida como nueva 
forma de adaptación de las estrategias tradicionales contrainsurgentes. UNISCI Journal, 44, 1-18. http://dx.doi. org/10.5209/RUNI.55778

Ramos, N. L., \& Cordoba, R. (2007). La contribución del proceso de paz a la construcción de la democracia en El Salvador (1992-2004). Construyendo la democracia en sociedades posconflicto. http://www.idrc.ca/EN/Resources/Publica tions/openebooks/340-9/index.html

Reyes, M. (2016). Desafíos de la inteligencia militar del ejército nacional en un escenario de posconflicto [Trabajo de grado de especialización no publicado]. Universidad Militar Nueva Granada.

Rojas, J. (2016). Etapas del conflicto armado en Colombia: hacia el posconflicto. Latinoamérica: revista de estudios latinoamericanos, 62, 227-257. https://doi.org/10.1016/j. larev.2016.06.010

Sainz, J. (2012). Inteligencia Táctica. UNISCI Discussion Papers.

Sancho, C. (2018). Ciberinteligencia: contextualización, aproximación conceptual, características y desafíos. Centro de Investigaciones y Estudios Estratégicos. https://www.
anepe.cl/wp-content/uploads/Cuaderno-Trabajo-N\% C2\%B01-2018.pdf

Spencer, D., \& Gómez, J. (2011). Colombia: camino a la recuperación: seguridad y gobernabilidad 1982-2010. http://di gitalndulibrary.ndu.edu/cdm/ref/collection/chdspubs/ id/18089

Strong F. J., \& Jaramillo J. M. (2014). La Fuerza Aérea Colombiana y la acción integral, doctrina y política de Estado.

Torres, C. (2016). Conflictos territoriales y acuerdos de paz en Colombia, impactos en el ordenamiento territorial. Bitácora Urbano Territorial, 26(2), 7-10. http://dx.doi. org/10.15446/bitacora.v26n2.59304

Urban, M. (1992). Big Boys' Rules: The SAS and the Secret Struggle against the IRA. Faber and Faber.

Velasco et al. (2012). Inteligencia y seguridad: revista de análisis y prospectiva (n. 12).

Villarraga, A. (2017). Acuerdos de paz entre el estado y las guerrillas (1982 - 2017...). Corporación Viva la Ciudadanía. http://viva.org.co/cajavirtual/svc0531/pdfs/Articulo056 _531.pdf 